\title{
Le prix Architecture de bibliothèques et de centres d'archives du Québec
}

\section{The Prix Architecture de bibliothèques et de centres d'archives du Québec}

\section{El premio Arquitectura de bibliotecas y de centros de archivos de Quebec}

\section{Yvon-André Lacroix}

Volume 60, numéro 2-3, avril-septembre 2014

Bibliothèques et architecture

URI : https://id.erudit.org/iderudit/1025510ar

DOI : https://doi.org/10.7202/1025510ar

Aller au sommaire du numéro

Éditeur(s)

Association pour l'avancement des sciences et des techniques de la documentation (ASTED)

ISSN

0315-2340 (imprimé)

2291-8949 (numérique)

Découvrir la revue

Citer cet article

Lacroix, Y.-A. (2014). Le prix Architecture de bibliothèques et de centres d'archives du Québec. Documentation et bibliothèques, 60(2-3), 62-73. https://doi.org/10.7202/1025510ar
Résumé de l'article

Le prix Architecture de bibliothèques et de centres d'archives du Québec est un prix bisannuel qui a été créé par le Congrès des milieux documentaires du Québec en 2011. Cet article retrace l'origine du prix en 2010, la mise en place des règlements et, pour les années 2011 et 2013, la formation du jury, la remise du prix et d'une mention honorifique. Chacune des sept candidatures de 2011 et des neuf candidatures de 2013 est illustrée d'une photographie. Les commentaires du jury de 2011 expriment les qualités de la lauréate du prix 2011, la Bibliothèque Raymond-Lévesque de la Ville de Longueuil, ainsi que celles de la mention accordée à la Bibliothèque de l'Université Laval. Quant à eux, les commentaires du jury du prix 2013 expriment les qualités de la lauréate de 2013, la Bibliothèque Laure Conan, de la Ville de La Malbaie, ainsi que celles de la mention accordée à la Bibliothèque Memphrémagog de la Ville de Magog.
Tous droits réservés (C) Association pour l'avancement des sciences et des techniques de la documentation (ASTED), 2014
Ce document est protégé par la loi sur le droit d'auteur. L'utilisation des services d'Érudit (y compris la reproduction) est assujettie à sa politique d'utilisation que vous pouvez consulter en ligne. 


\title{
Le prix Architecture de bibliothèques et de centres d'archives du Québec
}

\author{
YVON-ANDRÉ LACROIX \\ Consultant en bibliothéconomie et rédacteur \\ Directeur général de la diffusion de Bibliothèque \\ et Archives nationales du Québec (BAnQ) de 1999 à 2003 \\ yalacroix@gmail.com
}

\section{RÉSUMÉ | ABSTRACT | RESUMEN}

Le prix Architecture de bibliothèques et de centres d'archives $d u$ Québec est un prix bisannuel qui a été créé par le Congrès des milieux documentaires du Québec en 2011. Cet article retrace l'origine du prix en 2010, la mise en place des règlements et, pour les années 2011 et 2013, la formation du jury, la remise du prix et d'une mention honorifique. Chacune des sept candidatures de 2011 et des neuf candidatures de 2013 est illustrée d'une photographie. Les commentaires du jury de 2011 expriment les qualités de la lauréate du prix 2011, la Bibliothèque Raymond-Lévesque de la Ville de Longueuil, ainsi que celles de la mention accordée à la Bibliothèque de l'Université Laval. Quant à eux, les commentaires du jury du prix 2013 expriment les qualités de la lauréate de 2013, la Bibliothèque Laure Conan, de la Ville de La Malbaie, ainsi que celles de la mention accordée à la Bibliothèque Memphrémagog de la Ville de Magog.

\section{The Prix Architecture de bibliothèques et de centres d'archives du Québec}

Awarded biennially, the Prix Architecture de bibliothèques et de centres d'archives $d u$ Québec was created in 2011 by the Congrès des milieux documentaires du Québec. This article outlines the history of the prize since its inception in 2010, the rules and regulations and, in 2011 and 2013, the creation of a jury, and the awarding of the prize and a special honorary mention. A photograph shows each of the seven candidates for 2011 and the nine candidates for 2013. The 2011 jury highlighted the qualities of the Raymond-Lévesque Library of Longueuil, winner of the 2011 prize, as well as those for a mention made for the library of the Université Laval. The jury's comments for the 2013 prize underscore the qualities of the 2013 winner, the LaureConan Library in La Malbaie, as well as those for a mention for the Memphrémagog Library in Magog.

\section{El premio Arquitectura de bibliotecas y de centros de archivos de Quebec}

El premio bienal Arquitectura de bibliotecas y de centros de archivos de Quebec fue creado por el Congreso de medios documentales de Quebec en 2011. Este artículo detalla los orígenes del premio y la implementación de sus reglamentos en 2010, y describe la posterior composición del jurado, así como la entrega de un premio y de una mención de honor en los años 2011 y 2013. Una fotografía ilustra cada una de las siete candidaturas de 2011 y de las nueve candidaturas de 2013. Los comentarios del jurado de 2011 hacen referencia a las cualidades del candidato galardonado ese mismo año, la Biblioteca Raymond-Lévesque de la ciudad de Longueuil, así como de la Biblioteca de la Universidad Laval, que recibió una mención especial. Por su parte, los comentarios del jurado del premio 2013 reflejan las cualidades de la ganadora de ese mismo año, la Biblioteca Laure-Conan de la ciudad de La Malbaie, así como de la Biblioteca Memphrémagog de la ciudad de Magog, que recibió una mención especial.

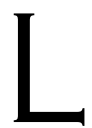

E PRIX ARChitecture DE BIBLIOThÈQUeS ET DE centres d'archives du Québec a été créé par le Congrès des milieux documentaires du Québec (CMD), qui émane d'un regroupement de neuf associations professionnelles des milieux des archives et des bibliothèques du Québec. La création de ce prix vise à donner une visibilité et un rayonnement aux réalisations architecturales de bibliothèques et de centres d'archives du Québec et à reconnaître l'amélioration de l'offre de services de ces lieux de vie et de mémoire. Le prix est accordé aux deux partenaires d'un projet, soit le client/bibliothèque et le maître d'ouvrage/architecte. Il souligne ainsi la collaboration indispensable des milieux documentaires et architecturaux à la réalisation d'une œuvre de qualité.

\section{Chronologie de la création du prix}

À l'hiver 2010, Guylaine Beaudry, alors présidente de la Corporation des bibliothécaires professionnels du Québec (CBPQ) et présidente du comité organisateur du CMD, estime que depuis plusieurs années, le Québec s'illustre par la qualité architecturale de ses bibliothèques et de ses centres d'archives. Puisque j'ai déjà écrit un article sur ce sujet ${ }^{1}$, elle m'approche et me consulte sur la possibilité de créer un prix en architecture de bibliothèques et de centres d'archives du Québec, un peu à l'instar de ce qui se fait déjà dans d'autres milieux bibliothéconomiques, particulièrement en Amérique du Nord.

Lors de la réunion du 30 mars 2010 du comité organisateur du CMD, Guylaine Beaudry propose la création d'un "prix Nouvelle Construction/Rénovation majeure ", qui "pourrait être décerné pour la première fois en 2011 " lors du congrès du CMD. Les membres me désignent président du comité de ce prix. Ils me demandent "de préparer une version de travail des règlements" et "souhaitent que ce prix ait un rayonnement plus large que les seuls milieux documentaires $"^{2}$.

1. Lacroix, Yvon-André. 2008. Quand les bibliothèques concourent et séduisent les architectes. Argus \& Documentation et bibliothèques (37) 1, 117-127.

2. Compte rendu de la réunion du 30 mars 2010 du comité organisateur du Congrès des milieux documentaires du Québec [Document non publié]. 
Ils me demandent également d'établir une grille d'analyse et d'évaluation, de prioriser l'efficacité fonctionnelle ou organisationnelle des projets soumis, puis les aspects esthétiques et architecturaux ainsi que l'implication de la communauté et des bibliothécaires ou des archivistes $^{3}$.

Je consulte alors les règles de participation et de présentation des prix d'excellence en architecture remis par divers organismes, tant sur les plans de la bibliothéconomie et de l'architecture, notamment :

- le Prix d'excellence Gérard-Desrosiers en aménagement de bibliothèque du Réseau BIBLIO du Québec;

- les Library Building Awards de l'Ontario Library Association (OLA);

- les Public Library Building Awards de la Chartered Institute of Library and Information Professionals (CILIP);

- les Prix d'excellence en architecture de l'Ordre des architectes du Québec (OAQ);

- les Prix d'excellence en architecture de l'Institut royal d'architecture du Canada (IRAC);

- les Awards of excellence de la revue Canadian Architect.

La rédaction d'une version quasi définitive des règlements est prête à la fin de l'été 2010. En octobre, une rencontre est tenue avec Jean-Pierre Dumont, directeur général et secrétaire de l'Ordre des architectes du Québec (OAQ), pour une discussion autour des règlements et de la collaboration de l'OAQ à ce projet de prix. Un mois plus tard, monsieur Dumont confirme "que le [conseil exécutif] de l'OAQ a accepté de collaborer à l'événement 2011 du prix Architecture de bibliothèques et de centres d'archives. Il félicite et remercie ses organisateurs pour cette heureuse initiative qui contribue au rayonnement de l'architecture au Québec. La collaboration de l'Ordre prendra notamment la forme d'une diffusion auprès de ses membres lors du lancement $d u$ prix et lors de l'annonce des lauréats ${ }^{4}$. Lors de la tenue du Congrès des milieux documentaires de novembre 2010, la création du prix Architecture de bibliothèques et de centres d'archives du Québec est annoncée officiellement, simultanément à la création du prix Innovation.

En janvier 2011 est choisi le thème du troisième Congrès des milieux documentaires. Intitulé "Redéfinir l'espace documentaire ", il porte sur l'architecture et l'aménagement des bibliothèques. Les règlements du prix Architecture sont acceptés et la présidence du jury m'est confiée. Avant les vacances de l'été, le CMD procède à la nomination des membres du jury, à la promotion et au lancement du prix Architecture 2011, ainsi qu'à la création d'un trophée par la firme RITA design (voir encadré, p. 73).

Le jury du prix Architecture 2011 se réunit le 4 novembre pour étudier les sept candidatures reçues. Le 30 novembre a lieu la cérémonie de la remise du prix Architecture 2011 ainsi que d'une mention honorifique.

Le jury du prix Architecture 2013 se réunit le 6 novembre 2013 pour étudier les neuf candidatures reçues. Le 25 novembre a lieu la cérémonie de la remise du prix Architecture 2013 ainsi que d'une mention honorifique.

\section{À propos du Congrès des milieux documentaires du Québec (CMD)}

Le Congrès des milieux documentaires du Québec est organisé depuis 2009 par les neuf associations qui composent les milieux documentaires du Québec : l'Association des archivistes du Québec (AAQ), l'Association pour l'avancement des sciences et des techniques de la documentation (ASTED), la Corporation des bibliothécaires professionnels du Québec (CBPQ), l'Association des bibliothécaires du Québec/Quebec Library Association (ABQLA), l'Association pour la promotion des services documentaires scolaires (APSDS), l'Association professionnelle des techniciennes et des techniciens en documentation du Québec (APTDQ), l'Association des bibliothèques publiques du Québec (ABPQ), le Réseau BIBLIO du Québec et la Special Libraries Association (SLA) - Section de l'Est du Canada. En regroupant plus de 1 ooo participants, le Congrès des milieux documentaires du Québec constitue l'événement annuel le plus important de la communauté des bibliothécaires, des archivistes et des techniciens en documentation. Le Congrès des milieux documentaires est le rendez-vous annuel principal pour les professionnels québécois des milieux documentaires.
3. Compte rendu de la réunion du 20 septembre 2010 du comité organisateur du Congrès des milieux documentaires du Québec [Document non publié].

4. Courriel de Jean-Pierre Dumont à Yvon-André Lacroix avec copie conforme à Guylaine Beaudry, Pierre Frisko et Christine Daffe, 11 novembre 2010. 


\section{Les règlements du prix ${ }^{5}$}

\section{Admissibilité}

Les participants doivent respecter obligatoirement les cinq règles d'admissibilité suivantes :

1. Le prix est ouvert à toutes les réalisations d'architecture de bibliothèques et de centres d'archives du Québec. Une institution ou un service, seul ou en partenariat avec d'autres, peut présenter une candidature par projet. Les types de bibliothèques ou centres d'archives suivant sont admissibles :

- les bibliothèques publiques;

- les bibliothèques académiques (universitaires et collégiales);

- les bibliothèques scolaires (primaires et secondaires);

- les bibliothèques institutionnelles, spéciales et privées permettant l'accès au public;

- les centres d'archives publics ou privés permettant l'accès au public.

2. Le prix est attribué pour un bâtiment neuf, un agrandissement, une rénovation, un recyclage, une conservation ou une restauration patrimoniale.

3. Les bibliothèques et centres d'archives doivent être localisés sur le territoire du Québec.

4. Les travaux doivent être complétés et l'ouverture au public des bibliothèques et des centres d'archives doit avoir eu lieu selon les dates suivantes :

- pour le prix Architecture 2011, entre le $1^{\mathrm{er}}$ juillet 2008 et le 30 juin 2011;

- pour le prix Architecture 2013, entre le $1^{\text {er }}$ juillet 2011 et le 30 juin 2013.

5. La candidature doit être déposée avec l'accord de l'architecte et du donneur d'ouvrage du bâtiment.

\section{Critère d'analyse global}

Le jury tient globalement compte des critères d'analyse suivants, soit une approche innovante et créative de la part de l'architecte et une intégration exemplaire d'une bibliothèque ou d'un centre d'archives dans son milieu, grâce au recours à des solutions fonctionnelles et innovantes favorisant une appropriation conviviale, tant de la part des usagers que du personnel, et une nette amélioration de l'offre de services.

Le partenariat et l'implication avec le milieu ainsi que les critères artistiques et esthétiques sont également considérés. Par ailleurs, le jury évalue l'innovation, la performance et la conformité du projet avec les principes du développement durable, l'accessibilité universelle du

5. Les règlements du prix et tous les renseignements utiles sont accessibles à : $<$ https://www.milieuxdoc.ca/2013/congres-des-milieux-documentaires-liste. php?id=14438\&b_programme $=>$. lieu et la qualité de l'intégration urbaine et sociale de celui-ci. Il prend également en compte d'autres disciplines telles que l'ingénierie, les arts et l'aménagement paysager.

\section{Le jury du prix}

Le jury responsable de l'attribution du prix Architecture de bibliothèques et de centres d'archives du Québec est composé de sept membres bénévoles, dont cinq membres qui sont issus des milieux documentaires (archivistes, bibliothécaires, techniciens en documentation), ainsi que deux représentants des milieux de l'architecture, du design ou des arts. Cette composition vise à permettre des échanges et des regards multidisciplinaires dans l'évaluation des candidatures reçues.

En 2011 et en 2013, le jury était constitué de sept membres, dont cinq en provenance de Montréal et deux de Québec, tous nommés par le comité organisateur du Congrès. Il s'agissait de :

- Marc Beaudoin, archiviste

- Yvon-André Lacroix, bibliothécaire et archiviste, président du jury

- Patricia Lemieux, technicienne en documentation et bibliothécaire

- Philippe Lupien, architecte

- Jacques Plante, architecte

- Diane Polnicky, bibliothécaire

- Claudette Poulin, bibliothécaire

Pour faciliter l'examen des dossiers de candidature, chaque membre reçoit un Cahier du jury. Celui-ci comprend notamment les règlements du prix, la liste des bibliothèques et centres d'archives candidats avec copie $\mathrm{CD}$ des photos en basse définition ainsi que les critères d'analyse. Pour chacun des candidats sont fournis le formulaire de dépôt de candidature, une capsule de 150 mots, un texte descriptif de 1 ooo mots de même que les plans d'implantation et d'aménagement, les coupes et les élévations.

Le jury jouit d'une totale autonomie et procède en toute liberté aux délibérations pour désigner le lauréat du prix. Sa décision est finale et sans appel. Il requiert les services bénévoles de Me Ghislain Roussel, avocat conseil, secrétaire général et directeur des affaires juridiques de Bibliothèque et Archives nationales du Québec de 1998 à 2009. Tous les membres signent le formulaire Engagement de confidentialité des délibérations $d u$ jury et déclaration de conflits d'intérêts, acceptent les règlements du prix de même que les neuf critères d'évaluation retenus, et vérifient l'admissibilité et la conformité des pièces jointes aux dossiers de candidature. Le jury procède ensuite à l'évaluation des dossiers et profite du déjeuner sur place en vue de planifier la suite de la sélection. 
Les constats des membres sont notés pour une première rédaction qui servira à faire l'éloge du lauréat du prix, et du lauréat d'une mention s'il y a lieu. À la fin des délibérations, tous les membres du jury remettent au secrétaire du jury leur exemplaire de tous les dossiers de candidature, ainsi que toutes leurs notes personnelles.

\section{Le lauréat du prix}

Le lauréat, à la fois la bibliothèque et le ou les architectes, est proclamé lors d'une cérémonie dans le cadre du Congrès des milieux documentaires, tenue au Palais des congrès de Montréal. Le jury se réserve le droit d'accorder ou non le prix, et de décerner une mention pour un projet remarquable. Le lauréat reçoit un trophée et l'attribution du prix est communiquée tant auprès des grands médias qu'auprès des médias professionnels des milieux bibliothéconomiques, archivistiques et architecturaux. Le lauréat jouit aussi d'une diffusion sur place tant par un panneau/affiche que par une projection en boucle d'une vingtaine de photographies de chacune des candidatures, dont les siennes.

\section{Conclusion}

Deux étapes viennent d'être franchies par les remises du prix Architecture en 2011 et en 2013. Cette relation interprofessionnelle hautement qualitative avec les architectes est une belle promesse pour le futur de l'architecture des bibliothèques et des centres d'archives au Québec. Des lieux de plus en plus inspirants ne peuvent que contribuer de belle façon au progrès de la connaissance et de la vie culturelle du Québec. C'est même une occasion exceptionnelle de stimuler et de valoriser l'ensemble des milieux documentaires, qui en sont d'ailleurs les premiers grands gagnants. En effet, en plus de l'intérêt des architectes et des ingénieurs, une telle démarche attire sur nos professions des regards positifs et reconnaissants, de la part notamment de nos gestionnaires et de nos bailleurs de fonds, de nos clients et de nos usagers, réels et potentiels, ainsi que de tous les Québécois.

En terminant, nos remerciements s'adressent aux membres et au secrétaire du jury pour leur généreux et professionnel bénévolat, aux directeurs des bibliothèques et aux firmes d'architectes pour leur participation enthousiaste, à l'Ordre des architectes du Québec et à sa présidente, Nathalie Dion, pour leur collaboration. Tous les responsables des neuf associations du Congrès des milieux documentaires et du comité organisateur méritent toute notre considération, à la fois pour leur ferme volonté de témoigner de leur force associative et pour leur esprit d'ouverture et de pluridisciplinarité.

\section{Les candidatures du prix Architecture 2011}

En 2011, le prix Architecture a été remis pour des projets réalisés entre le $1^{\mathrm{er}}$ juillet 2008 et le 30 juin 2011. Le jury a étudié sept dossiers de bibliothèques auxquels ont participé quatre firmes d'architectes et trois consortiums d'architectes :

\section{7 candidatures - par types de bâtiments}

- Bibliothèques

- bâtiment neuf : 3

- agrandissement : 2

- agrandissement et rénovation majeure : 1

- rénovation majeure : 1

- Centres d'archives

- aucun

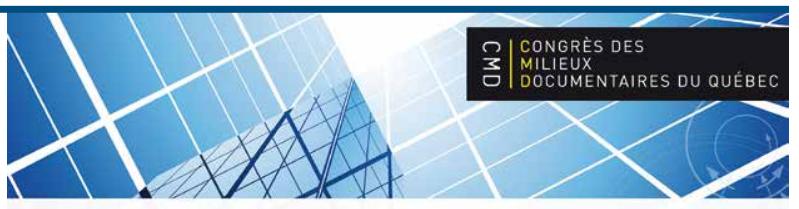

Prix Architecture 2011

de bibliothèques et de centres d'archives du Québec

\section{7 candidatures - par types de bibliothèques}

- 5 bibliothèques municipales de la région métropolitaine de Montréal

- 4 de la Rive-Sud

- 1 de la Rive-Nord

- 1 bibliothèque de niveau collégial

- 1 bibliothèque de niveau universitaire 


\section{Les candidatures du prix Architecture 2011}

$\begin{aligned} & \text { Ville de Boucherville - Bibliothèque Montarville-Boucher-De la Bruère } \\ & \text { Brière Gilbert Associés Architectes et Denis St-Louis Architecte } \\ & \text { (Agrandissement, septembre 2009) }\end{aligned}$
Photo: Christian Perreault
Ville de Longueuil - Arrondissement Saint-Hubert - Bibliothèque Raymond-Lévesque
Manon Asselin Architecte et Jodoin Lamarre Pratte Associés consortium
(Bâtiment neuf, janvier 2011)
Photo : Julien Perron-Gagné

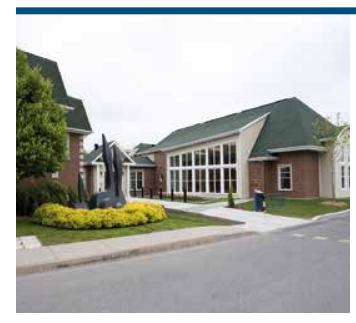

Ville de Notre-Dame-de-l'Île-Perrot - Bibliothèque Marie-Uguay

Poirier Fontaine Architectes

(Bâtiment neuf, octobre 2009)

Photo : Ville de Notre-Dame-de-l'Île-Perrot

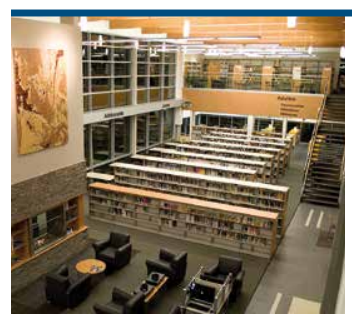

Ville de Sainte-Catherine - Bibliothèque municipale de Ville de Sainte-Catherine

Leclerc-Architectes

(Bâtiment neuf, novembre 2009)

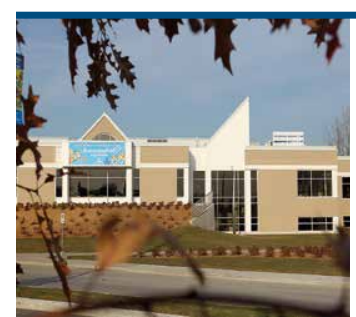

Photo : Judith Cailhier

Ville de Sainte-Thérèse - Bibliothèque de Sainte-Thérèse

Yves Woodrough Architectes

(Agrandissement et rénovation majeure, novembre 2010)

Photo : André Préard

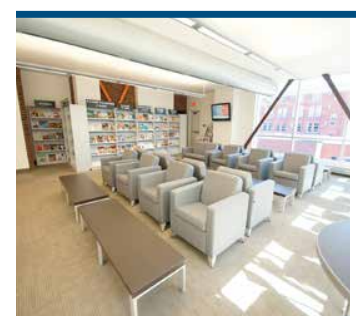

Cégep de Granby-Haute-Yamaska

Bibliothèque et technologies éducatives du Cégep de Granby-Haute-Yamaska

Lussier Architecte

(Agrandissement, mai 2011)

Photo : Pascal Rameux

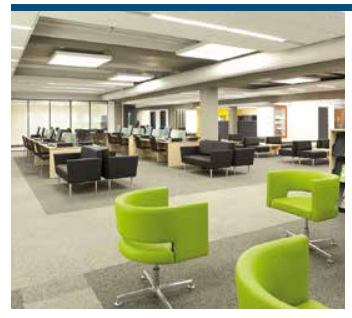

Université Laval - Service des immeubles - Pavillon Jean-Charles-Bonenfant Bibliothèque des sciences humaines et sociales - $4^{e}$ étage

Consortium Bélanger, Beauchemin, Morency et Anne Carrier, Architectes

(Rénovation majeure, mars 2011)

Photo : Claude Mathieu 


\section{Le lauréat du prix Architecture $2011^{6}$}

Le jury accorde à l'unanimité le prix

Architecture 2011 aux deux partenaires :

Ville de Longueuil - Bibliothèque Raymond-

Lévesque et à Manon Asselin Architecte

et Jodoin Lamarre Pratte et associés, Architectes

en consortium, pour le projet de la Bibliothèque

Raymond-Lévesque de la Ville de Longueuil.

Le jury souligne l'intégration achevée des concepts de globalité architecturale et de fonctionnalité bibliothéconomique. Cette symbiose s'exprime par un dialogue permanent entre les espaces aussi nombreux que variés réservés aux usagers, aux services et aux collections. Dialogue entre l'art et l'esthétique, entre l'utilisation judicieuse tant du bois, du verre et du béton que du mobilier et de l'éclairage. Dialogue enfin entre le paysage et l'environnement, par les liens avec le boisé et le bassin minéral de rétention d'eau.

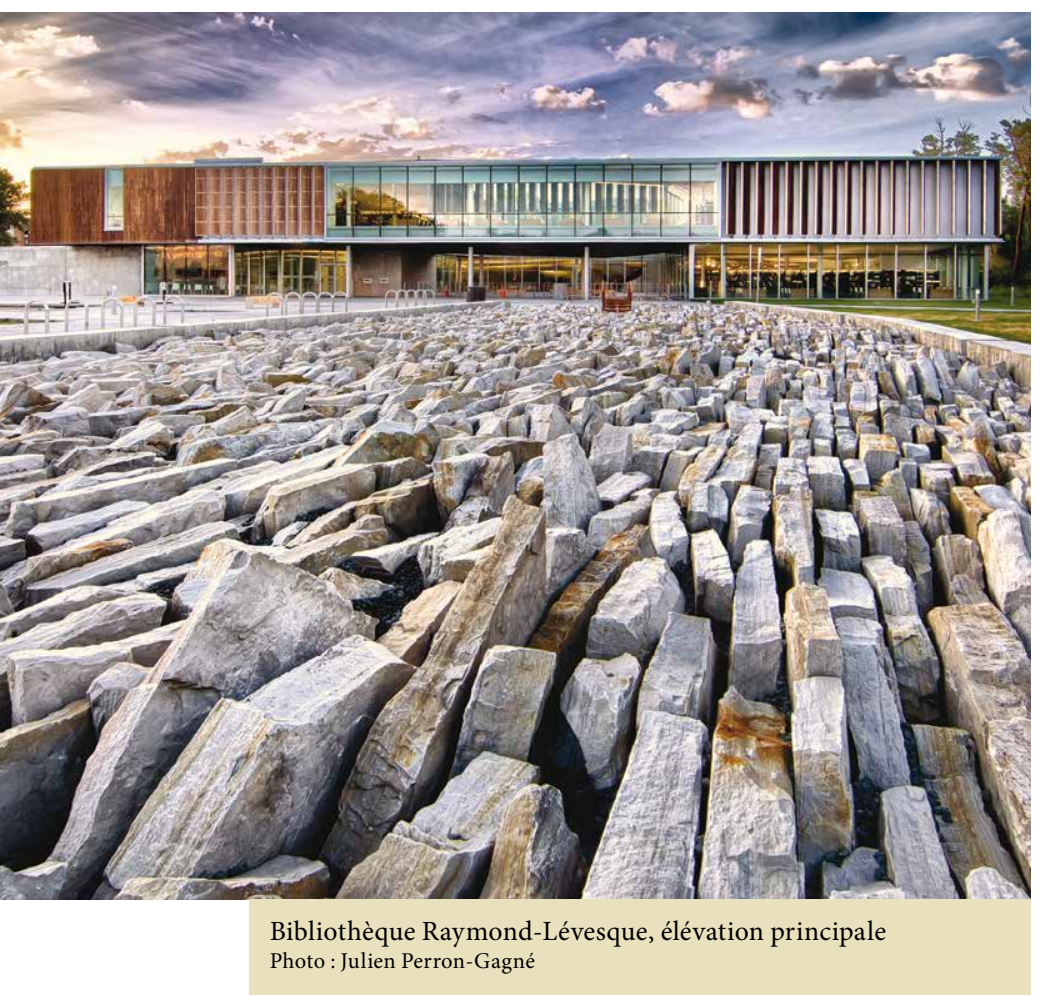

L'ambition des architectes de propulser la bibliothèque vers l'avenir est mission accomplie. Sa superficie presque carrée permet agrandissement et réaménagement et fait preuve de cohérence et de durabilité. Une bibliothèque facilement lisible, en transparence et sans aucune opacité, aussi belle de jour que belle de nuit, du dehors que du dedans.

6. Mentionnons que cette réalisation a reçu, depuis trois ans, plus d'une douzaine de prix dont, en novembre 2013, le Prix d'excellence en architecture de la catégorie « Bâtiments institutionnels » de l'Ordre des architectes du Québec et, en mars 2014, la Médaille du Gouverneur général en architecture.
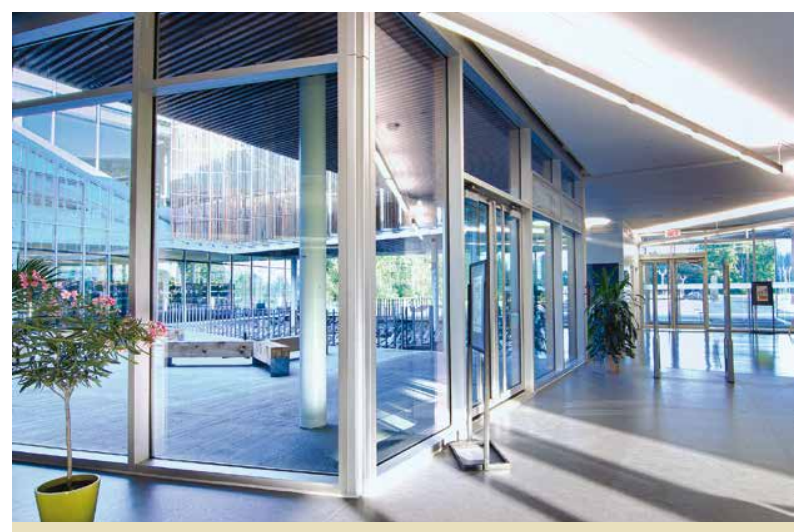

Bibliothèque Raymond-Lévesque, hall d'entrée

avec vue sur le préau

Photo : Julien Perron-Gagné

Les usagers s’approprient la bibliothèque par étapes, d'abord dès le stationnement, le parvis et le hall, puis grâce à un déploiement d'axes bien identifiés et d'espaces conviviaux, par l'emplacement des comptoirs de services et de l'escalier central, par la variabilité du traitement du plafond, de l'utilisation fort créative de la lumière artificielle, de la généreuse lumière naturelle offerte par une large fenestration en lien constant avec l'environnement et le préau. À la fois au centre du bâtiment et ouvert sur le bassin minéral, la forte présence de ce préau aux allures de cloître moderne génère calme et réflexion.

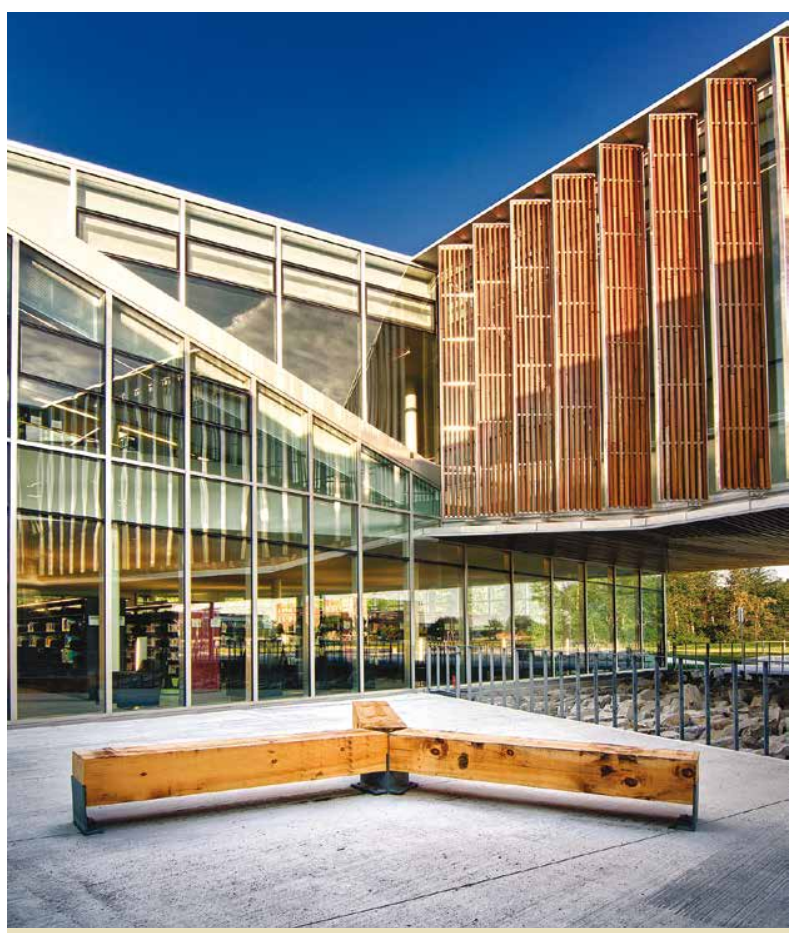

Bibliothèque Raymond-Lévesque, préau

Photo : Julien Perron-Gagné

Cette réalisation offre un parcours riche en découvertes, notamment le café Dewey et l'espace presse, la zone adolescent et l'espace mère-nourrisson. 


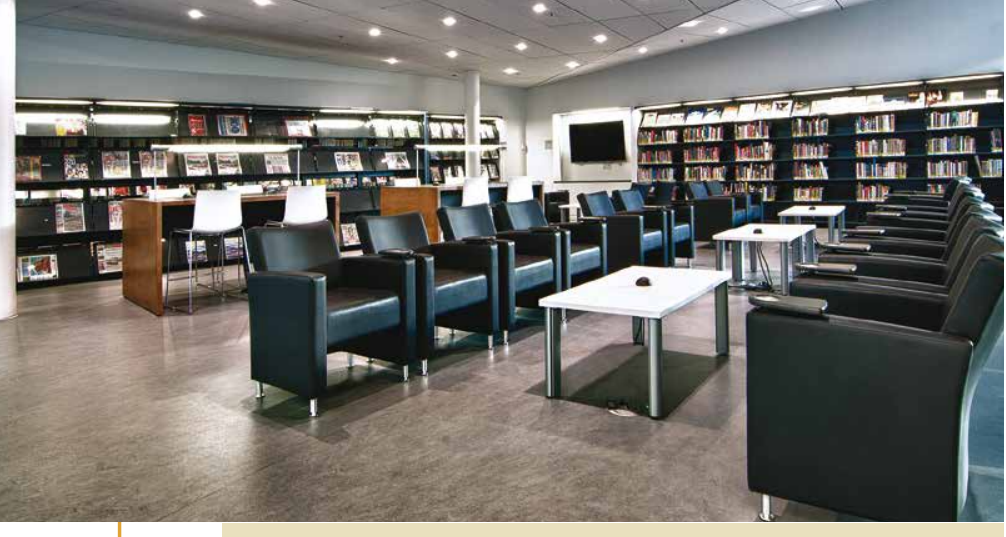

Bibliothèque Raymond-Lévesque, espace presse Photo : Julien Perron-Gagné

Le rayonnage présenté par endroit en diagonale crée une dynamique et adoucit le côté généralement rigide des bibliothèques. Les tables de travail de type réfectoire de la grande salle sont à la fois tendance et un clin d'œil au passé.

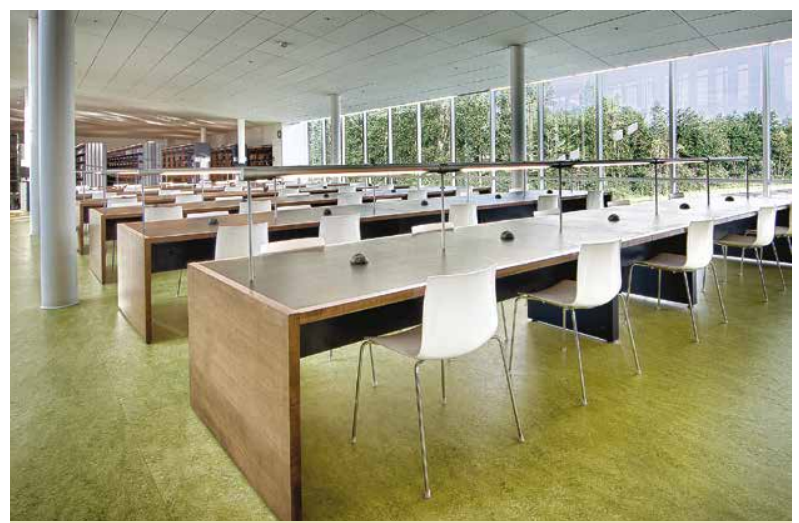

Bibliothèque Raymond-Lévesque, salle de travail

Photo : Julien Perron-Gagné

Le jury est unanime à reconnaître l'apport exceptionnel de cette bibliothèque à une juste valorisation des besoins et du bien-être des usagers et du personnel : un lieu de vie créateur de capital social.

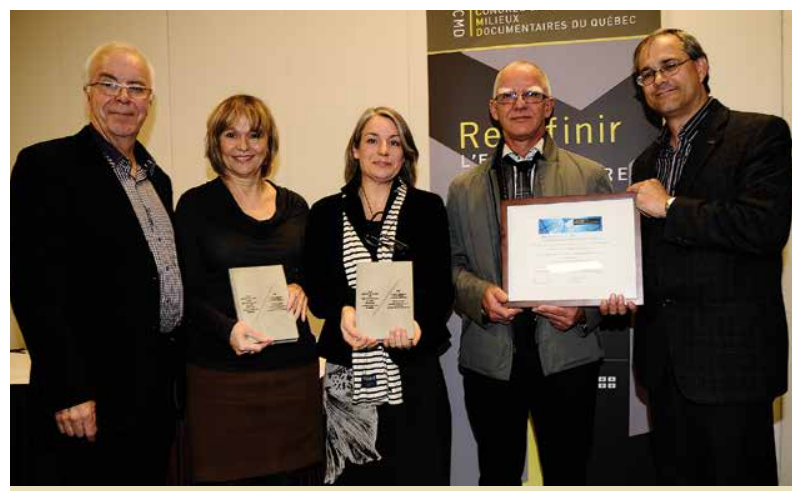

La Bibliothèque Raymond-Lévesque a reçu le prix Architecture 2011 à l'occasion du Congrès des milieux documentaires, le 30 novembre 2011. De gauche à droite : Yvon-André Lacroix, président du prix Architecture 2011; Micheline Perrault, directrice de la Bibliothèque Raymond-Lévesque; Manon Asselin, architecte; Marc Laurendeau, architecte associé principal de la firme Jodoin, Lamarre, Pratte; Pierre Martel, architecte, chef du service des bâtiments de la Ville de Longueuil. Photo : Renaud Sakelaris

\section{Une mention honorifique en 2011}

Le jury du prix Architecture 2011

accorde à l'unanimité une mention honorifique

aux deux partenaires : Bibliothèque de l'Université

Laval et Bélanger, Beauchemin, Morency Architectes et Urbaniste et Anne Carrier Architecte pour le projet de la Bibliothèque des sciences humaines et sociales de l'Université Laval, Pavillon Jean-Charles-Bonenfant, $4^{e}$ étage.

Le jury apprécie la très grande qualité du réaménagement des espaces et du renouvellement du mobilier du quatrième étage de la bibliothèque. Il note que c'est la même firme d'architectes en 2011 et lors de la conception de l'édifice original datant de 1969. Il se réjouit de la continuité des intentions conceptuelles de l'architecture. En effet, il reconnaitt le respect du vocabulaire architectural, l'adéquation et la cohérence entre l'édifice d'origine et le réaménagement actuel. Tout dans l'aménagement est judicieusement évocateur de la fin de la décennie 1960, notamment le choix des matériaux et des couleurs, du mobilier et du tapis.

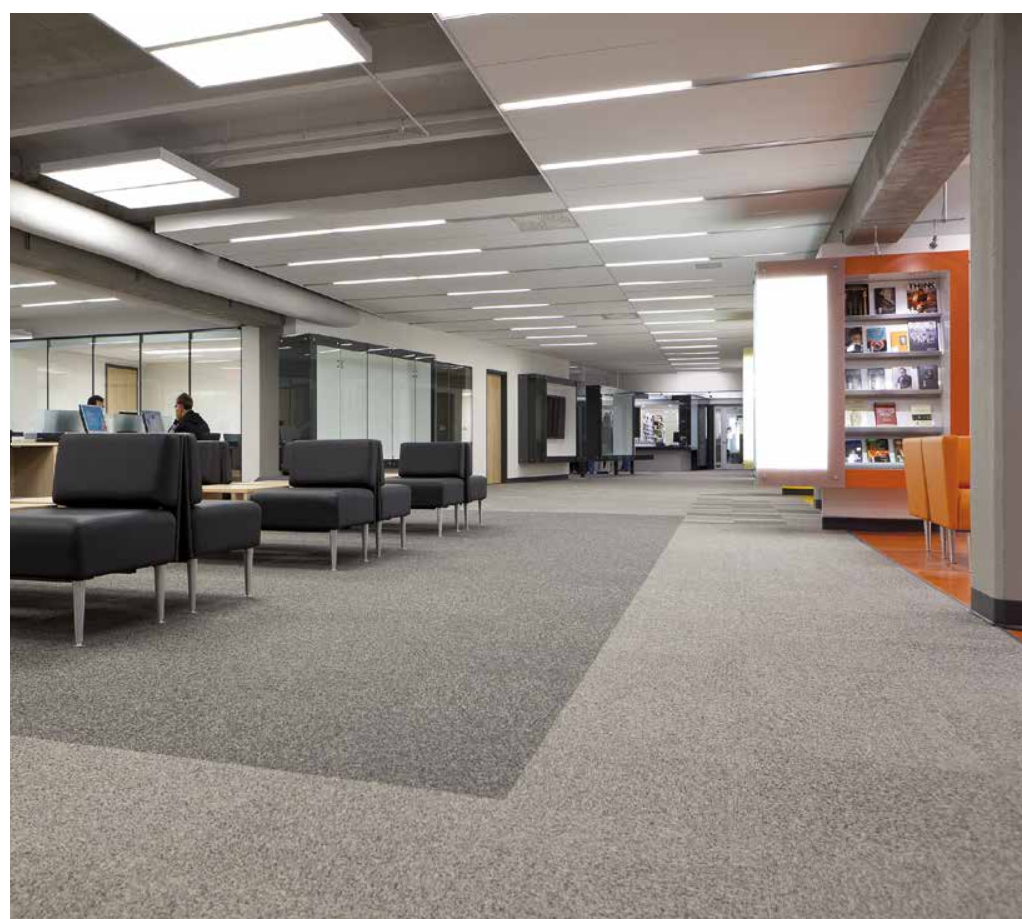

BSHS de l'Université Laval, espace de travail

Photo : Claude Mathieu

Le jury souligne le dégagement des espaces et du comptoir de référence, la luminosité naturelle, l'intervention dans le traitement du plafond et de l'éclairage artificiel ainsi que l'ouverture sur le boisé. Les lieux, autrefois aux airs d'entrepôt terne, se donnent maintenant des airs de lieux de vie qui respirent, qui facilitent la lisibilité et l'orientation en plus d'améliorer sensiblement la qualité de vie tant des usagers que du personnel. 


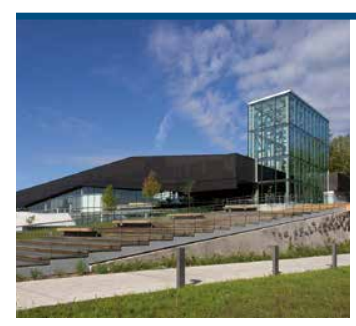

Ville de Montréal - Arrondissement Saint-Laurent - Bibliothèque du Boisé

Cardinal Hardy/Labonté Marcil/Éric Pelletier, architectes en consortium

(Bâtiment neuf)

Photo : Ville de Montréal. Arrondissement Saint-Laurent

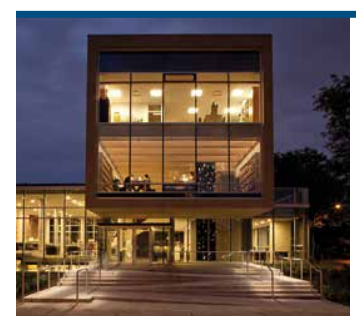

Ville de Mont-Royal - Bibliothèque Réginald-J.-P.-Dawson

Pierre Morency, architecte

(Agrandissement)

Photo : Normand Rajotte

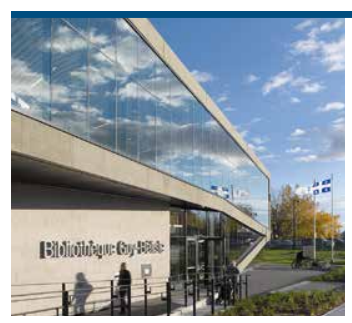

Ville de Saint-Eustache - Bibliothèque Guy-Bélisle

$\mathrm{ACDF}^{*}$ architecture

(Bâtiment neuf)

Photo : James Brittain

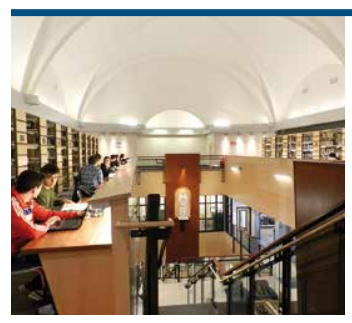

Cégep de Rimouski - Bibliothèque Gilles-Vigneault

Le Consortium d'architectes Goulet et LeBel + Proulx et Savard

(Rénovation majeure et restauration patrimoniale)

Photo : François Desjardins

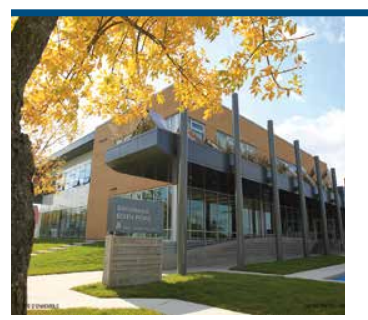

Commission scolaire des Navigateurs et Municipalité de Saint-Lambert-de-Lauzon

Bibliothèque municipale et scolaire - Bibliothèque Édith-Poirier

Larochelle et Desmeules, architectes

(Bâtiment neuf)

Photo : Pascal Ratthé

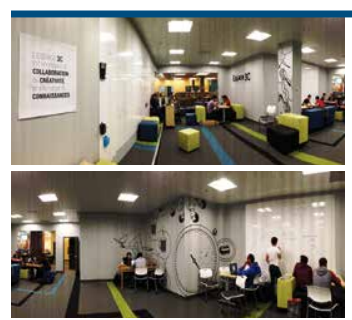

École de technologie supérieure - Bibliothèque

ESPACE TEMPS/Vincent Audette-Chapdelaine, conception, gestion de projet (Rénovation partielle)

Photo : École de technologie supérieure

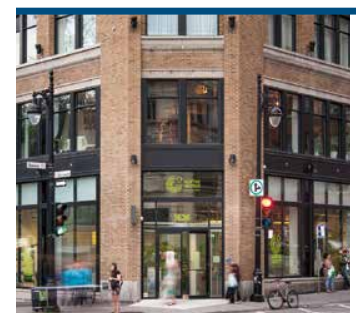

Goethe-Institut Montréal - Bibliothèque

Atelier TAUTEM

(Rénovation majeure et recyclage)

Photo : Jean-Guy Lambert 


\section{Le lauréat du prix Architecture 2013}

Le jury accorde à l'unanimité le prix

Architecture 2013 aux deux partenaires :

Ville de La Malbaie - Bibliothèque Laure-Conan

et Consortium Bisson/ACDF*/Desgagnés,

architectes (SENC) pour le projet

de la Bibliothèque Laure-Conan

de la Ville de La Malbaie.

Le jury a eu un coup de cœur pour ce bâtiment exceptionnel tant du point de vue de la bibliothéconomie que de l'architecture et de l'accessibilité, dont celle des personnes handicapées. Ce bâtiment fonctionnel a d'abord et avant tout été conçu pour les usagers. Ceux-ci ont une belle occasion de s'approprier la bibliothèque dès le hall d'entrée, grâce à un déploiement d'axes bien identifiés, d'espaces élégants et sobres, conviviaux et dégagés, lumineux et transparents, ainsi que par le positionnement du comptoir d'accueil.

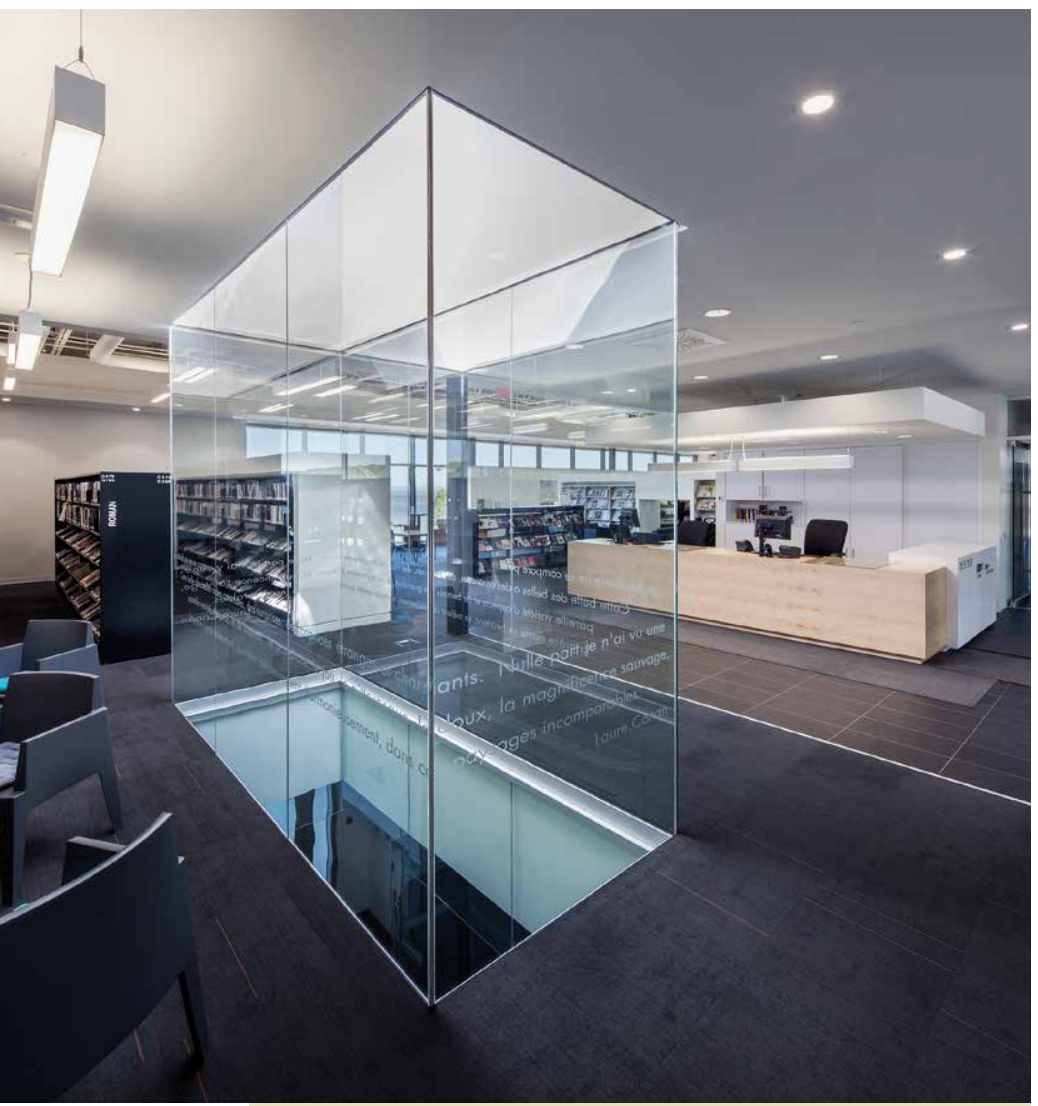

Bibliothèque Laure-Conan,

puits de lumière et comptoir d'accueil

Photo : Stéphane Groleau

Le jury apprécie le coin des enfants, la salle d'exposition et les espaces de travail pour les employés. Il est tout particulièrement ravi par la mise en valeur du paysage fluvial et montagneux, aussi omniprésent et majestueux que paisible. En un mot, ce lieu est une intime invitation aux citoyens et aux visiteurs de se réfugier dans les espaces intérieurs et les alcôves face au fleuve, comme sur les vérandas d'autrefois, et à s'y laisser happer à la fois par la lecture et par l'immensité.
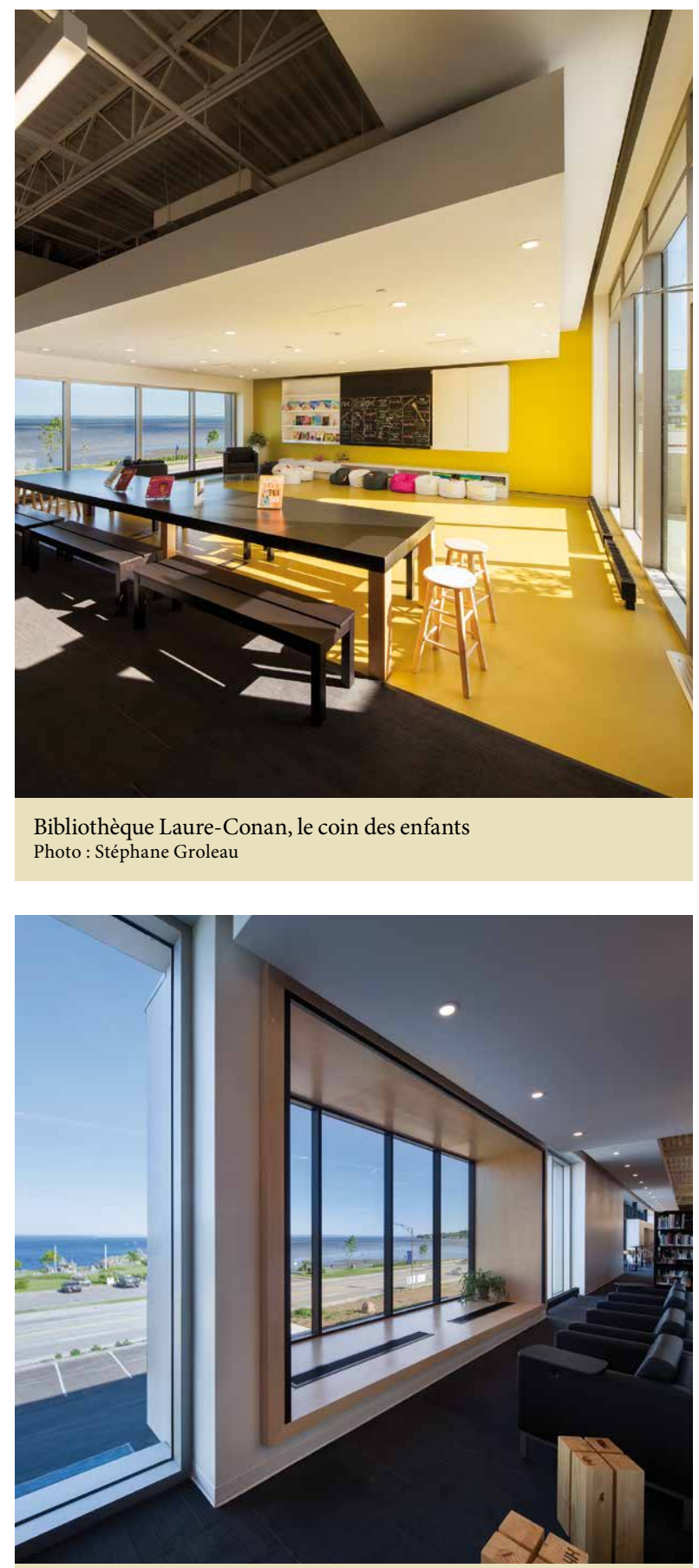

Bibliothèque Laure-Conan, la "véranda » avec ses fauteuils face au fleuve Saint-Laurent Photo : Stéphane Groleau

Le jury souligne également la réussite exemplaire de l'intégration de l'hôtel de ville et de la bibliothèque. Le bâtiment est le résultat d'une étude attentive de la topographie. En effet, sur deux paliers différents et accessibles en automobile, les deux fonctions, loin de se porter ombrage, se rehaussent mutuellement grâce 
à la réconciliation des deux accès. Le jury apprécie le lien étroit de la bibliothèque à la trame urbaine et à un environnement en développement, entre la ville et le fleuve. Le jury insiste sur le choix et la qualité des matériaux, dont des matériaux locaux tels que le bois, avec un rappel judicieux de la vocation maritime et forestière de la municipalité et de la région. Il est heureux de la manière sensible et délicate avec laquelle ont ainsi été réappropriées les berges du fleuve, un véritable baume à la suite du traumatisme causé par la route 362 .

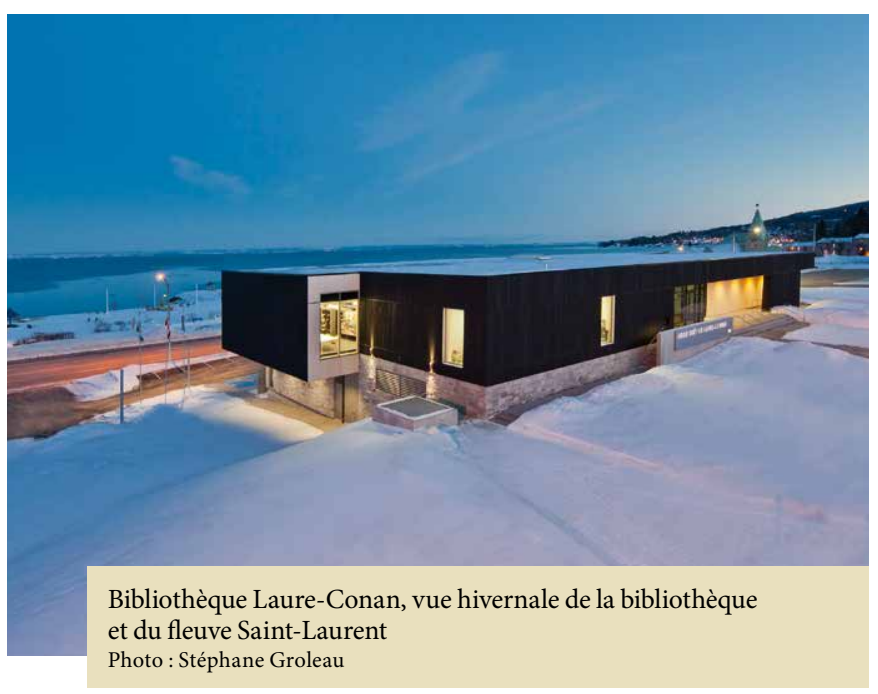

Le jury tient enfin à mentionner le signal fort donné par la réalisation de ce projet par le Conseil municipal d'une ville de 9 ooo habitants à l'égard d'un équipement culturel de qualité. Il s'agit d'un projet accompli avec délicatesse, malgré des contraintes budgétaires, un projet mobilisateur de toutes les forces locales afin de se doter d'un équipement moderne et de mettre également en valeur le patrimoine local et régional.

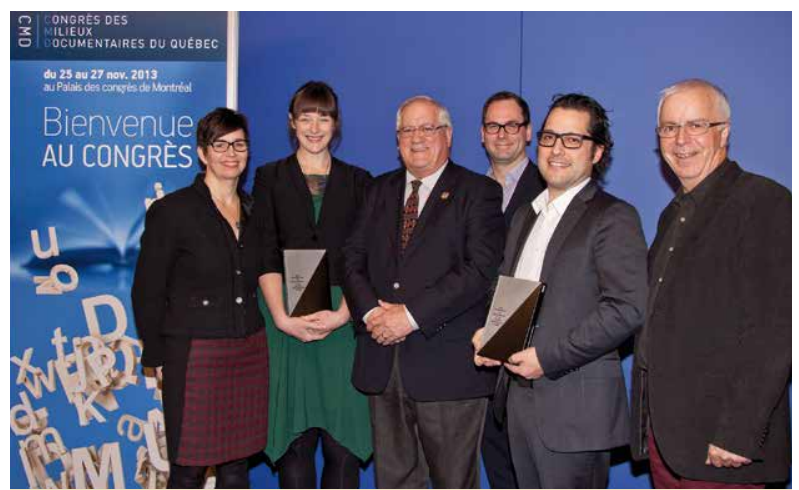

La Bibliothèque Laure-Conan a reçu le prix Architecture 2013 lors de la remise le 25 novembre 2013 du prix Architecture 2013 de bibliothèques et de centres d'archives du Québec.

De gauche à droite : Nathalie Dion, présidente de l'Ordre des architectes du Québec; Evelyn Bouchard, directrice de la Bibliothèque Laure-Conan; Fernand Charest, conseiller municipal de La Malbaie; Maxime Frappier, architecte; Jonathan Bisson, architecte; Yvon-André Lacroix, président du prix Architecture 2013

Photo : Martine Frigon

\section{Une mention honorifique en 2013}

Le jury du prix Architecture 2013 accorde à l'unanimité une mention honorifique aux deux partenaires: Ville de Magog - Bibliothèque Memphrémagog et ESPACE VITAL architecture pour le projet de la Bibliothèque Memphrémagog de la Ville de Magog.

Le jury estime qu'il s'agit ici d'un beau projet de restauration et de recyclage de l'église Sainte-Marguerite-Marie datant des années 1950, où l'on a réussi la mise en valeur d'une bibliothèque présentant de très beaux vitraux plutôt que celle d'une église contenant une bibliothèque.

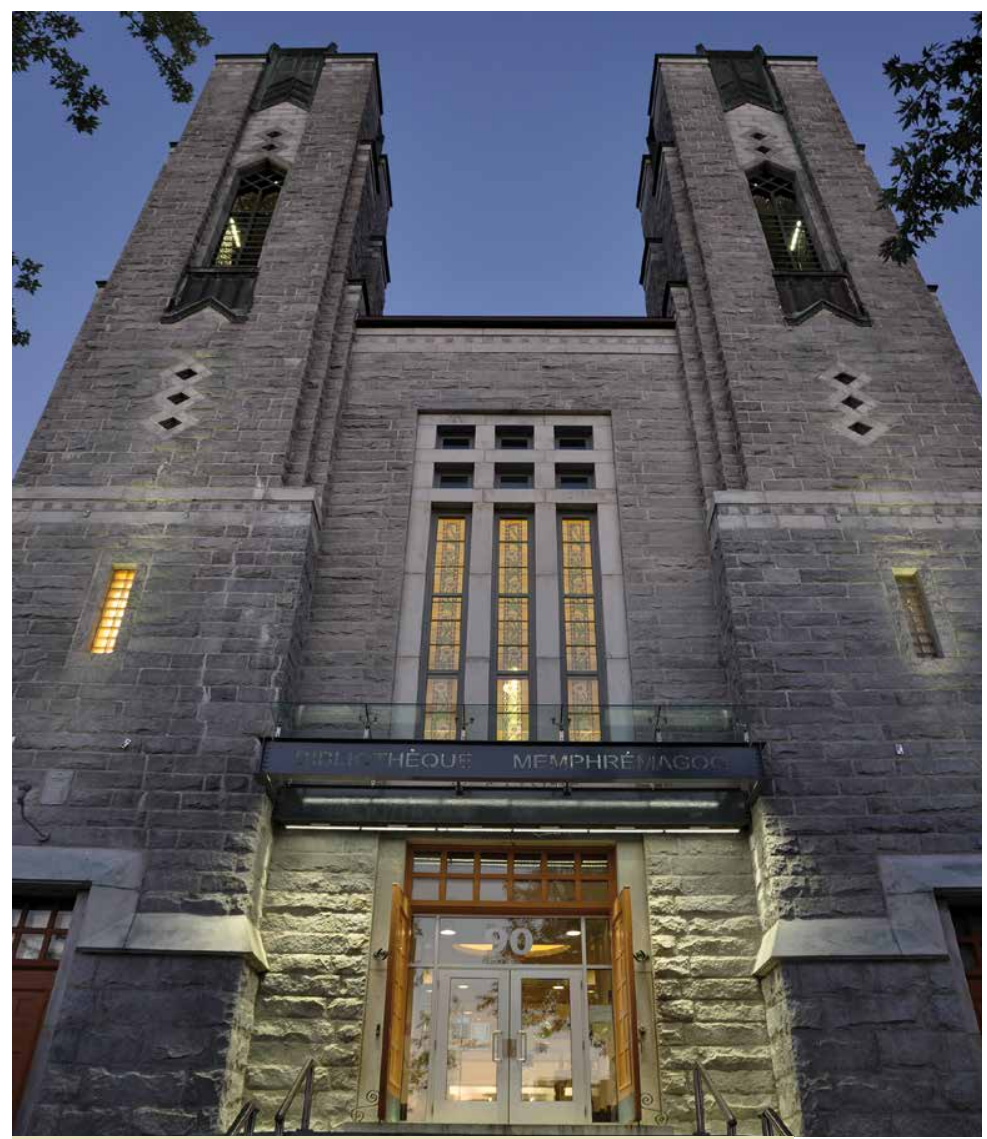

Bibliothèque Memphrémagog, vue extérieure

Photo : Stéphane Lemire

À l'intérieur du bâtiment, le jury relève l'ajout de planchers, l'éclairage suspendu, les percées, l'élégance de l'ameublement, la finesse, la transparence, la fraîcheur des lieux et la qualité particulière de la signalisation. Il note la volumétrie nouvelle, la conservation de l'axe du bâtiment, la sauvegarde du patrimoine religieux (l'extérieur, les portes en position d'ouverture et les vitraux), le soin apporté à la performance énergétique et à la sonorisation, la revitalisation d'un quartier ouvrier de Magog et le recyclage en un bâtiment fonctionnel, et ce, dans le respect de plusieurs contraintes, notamment patrimoniales. 

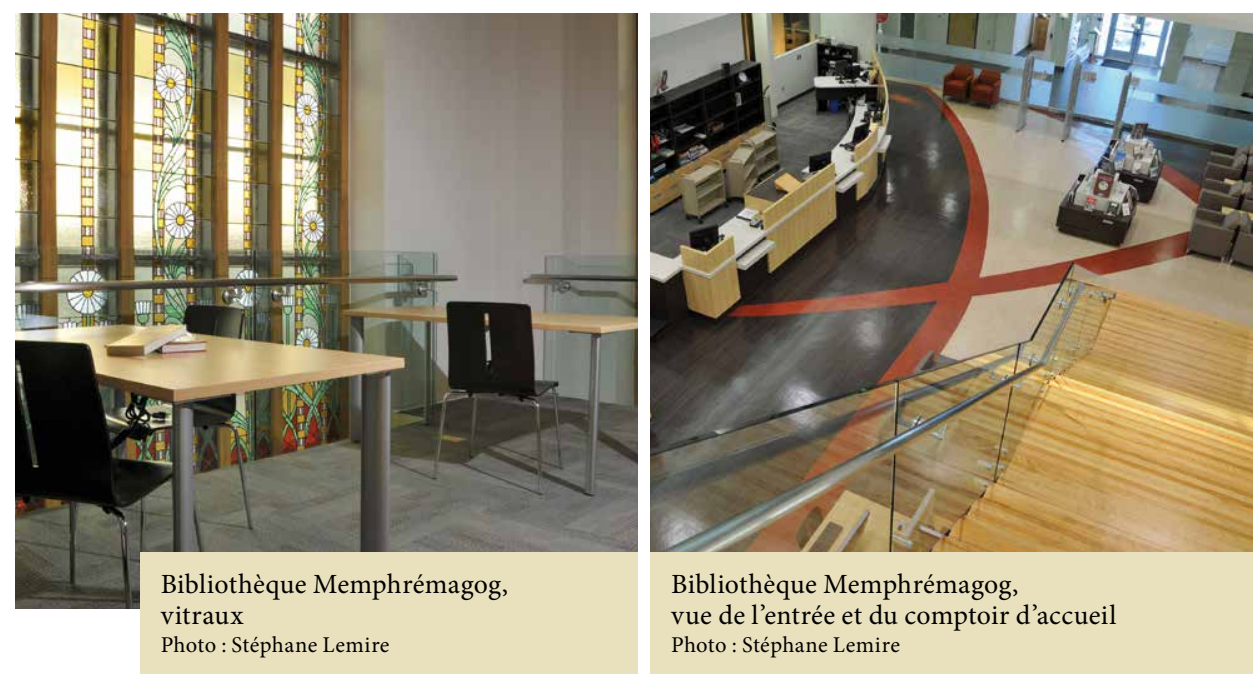

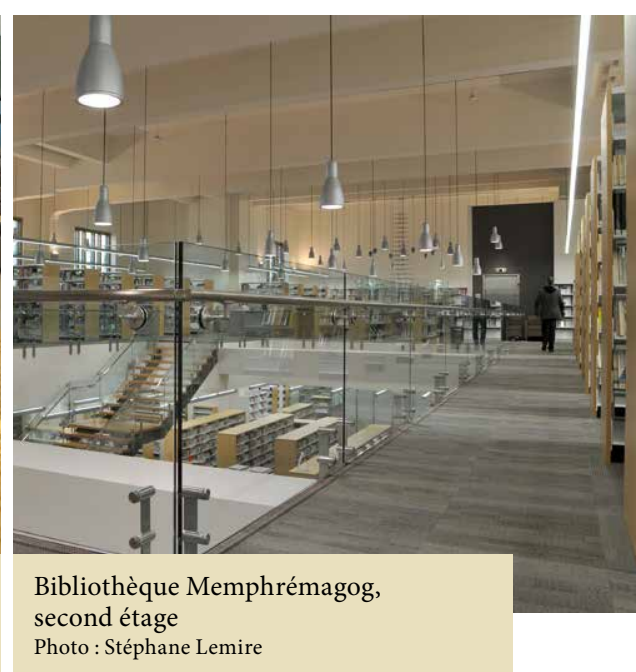

Le jury apprécie les espaces dédiés aux technologies de l'information, aucune perte d'espace, la récupération des dessous d'escaliers, le symbole des portes ouvertes, le rappel du parvis avec l'escalier extérieur, l'œuvre d'intégration à l'art et à l'architecture et le fait que la population ait été impliquée directement par un référendum concernant la nouvelle vocation de l'église.
Par cette reconnaissance, le jury souhaite sensibiliser d'autres bibliothèques et centres d'archives aux prises avec des lieux vétustes ou inappropriés en vue de profiter d'une éventuelle occasion permettant de recycler des édifices patrimoniaux qui répondent adéquatement aux besoins des clientèles d'aujourd'hui.

\section{Le trophée du prix Architecture du Congrès des milieux documentaires du Québec}

Créer le prix Architecture des bibliothèques et des centres d'archives du Québec comportait de concevoir son emblème, l'objet qui symboliserait le prix et distinguerait ses gagnants. Au printemps 2011, le comité organisateur du Congrès des milieux documentaires du Québec (CMD) a formé un comité composé de Francine Benoît-Plamondon, Régine Horinstein, Yvon-André Lacroix, Marcel Lajeunesse et Guylaine Beaudry dont le mandat était de faire concevoir et réaliser un trophée en vue de la première remise du Prix à l'automne 2011.

Quelques designers ont été contactés avant que le choix des membres du comité ne s'arrête sur le Studio Rita Design de Karine Corbeil et de Stéphane Halmaï-Voisard, qui avait déjà réalisé les trophées Art-Affaires de Montréal du Conseil des arts de Montréal ainsi que NUMIX, prix du Regroupement des producteurs multimédia (RPM). Dès juin 2011, les membres du comité ont rencontré l'équipe de Rita Design pour discuter du projet et lancer le développement de prototypes.
Il fallait un objet emblématique fort et qui ne souffrirait aucune ambiguïé. Après de longues discussions autour de plusieurs pistes, nous avons opté pour une certaine audace en choisissant une représentation d'un livre imprimé, au moment où nos bibliothèques entrent de plain pied dans la culture numérique. Le processus a mené à la création de l'objet par excellence des milieux documentaires : le livre.

Le trophée du prix Architecture est fait de béton, matière qui représente bien les constructions de nos bibliothèques du Québec. Sur sa surface cirée, au plat recto, sont inscrits en titre le nom du prix ainsi que le millésime et le nom du gagnant. Au plat verso, on peut lire le logo du CMD ainsi que la signature de Rita Design. Le nom du prix ainsi que le millésime sont repris au dos. L’objet, par l'utilisation du béton, est relativement lourd, on le sent bien présent lorsqu'on le tient entre les mains. Il est inséré dans un coffret fait d'aluminium peint en noir qui en couvre la moitié. Le coffret est chemisé de feutre à l'intérieur pour rendre plus agréable la manipulation des deux objets.

GUYLAINE BEAUDRY 